\title{
Tipps und Tricks für die problematische Metallentfernung von winkelstabilen Platten-Schrauben-Systemen
}

\author{
Dankward Höntzsch
}

\section{Zusammenfassung}

Die Metallentfernung kann bei winkelstabil verriegelten Schrauben in Platten bzw. internen Fixateuren in besonders gelagerten Fällen problematisch sein. Die hier geschilderten Tipps und Tricks kommen nicht alleine aus der Feder des Autors, sondern die Mitglieder der MIOEG stehen dahinter und sind als Koautoren zu verstehen.

\section{Tips and Tricks for the Difficult Removal of Metal in Angle-Stable Plate and Screw Systems}

In the case of angle-stable locking screws in plates or, respectively, internal fixation in specifically positioned cases, the removal of metal can be difficult. The tips and tricks described here are not solely derived from the author's own experience but rather originate from members of the MIOEG who should thus be considered as coauthors.

\section{Prävention}

Die Gründe für blockierte Schrauben oder ausgeleierte Schraubenantriebe sollen hier nicht weiter erörtert werden. Es sollen nur ein paar Anregungen gegeben werden, welche die Operateure bei der Implantation zur Prävention von Problemen für die Implantatentfernung beachten sollten.

- Verwende intakte Schraubendreher. Abgerundete Kanten der Schraubendreher können Grund für ein Durchdrehen sein. Dies beschädigt aber dann gleichzeitig die Flanken des Inbus.

- Wenn ein Inbus bei der Implantation beschädigt wird, sollte diese Schraube intraoperativ ausgetauscht werden! Aus Fairness gegenüber dem nächsten Operateur bei der Implantatentfernung und als Vorsorge für den Patienten, um Probleme bei der Entfernung der Schrauben möglichst zu vermeiden.

- Bei winkelstabilen Schrauben sollte das passende Drehmoment beachtet und der passende Drehmomentbe-

OP-JOURNAL 2012; 28: 170-173

(c) Georg Thieme Verlag KG Stuttgart · New York DOI http://dx.doi.org/10.1055/s-0032-1315296

grenzer (wenn vorgesehen) benutzt werden (Abb. 1).

- Vermeide falsche Angulationen bei winkelstabilen Schrauben, wenn sie nicht polyaxial ausgelegt sind. Die Verwendung der angebotenen Zielhilfen und die Beachtung der OP-Anleitung sind dringend zu empfehlen (Abb. 2).

- Verwende die richtige Anzahl von Schrauben am richtigen Platz. Zu wenig Schrauben werden überlastet, zu viele Schrauben am falschen Ort kosten bei der Implantation und Metallentfernung nur Zeit.

\section{Metallentfernung}

- Verwende von Anfang an nur intakte Schraubendreher. Überprüfe den Schraubendreher selbst. Ein abgenutzter Schraubendreher überträgt die Kraft nicht korrekt flächig. Beim Durchdrehen werden die Flanken des Inbus beschädigt. Dann hilft auch ein neuer intakter Schraubendreher nicht mehr. Dann ist es zu spät.

- Deshalb ist gerade für die Metallentfernung der beste Schraubendreher gerade gut genug.

- Der Inbus muss bis auf den Grund leer sein, damit der Schraubendreher in ganzer Länge eingeschoben werden kann.
Wenn der Inbus rundgedreht (ausgeleiert) ist (Abb. 3):

- Verwende eine konische, links schneidende Extraktionsschraube (Abb.4). Diese muss die richtige Größe in Durchmesser und Länge haben. Das Gewinde selbst muss unbeschädigt sein. Zwei Wege sind möglich. Der 1. und bevorzugt zu empfehlende:

a) Die konische, links schneidende Extraktionsschraube direkt in den beschädigten Inbus eindrehen.

b) Wenn dies nicht geht oder die Passung nicht genau stimmt, kann der Inbus knapp aufgebohrt werden (s.u.), ohne den Kopf abzutrennen. In das dann zylindrische Loch kann dann eine passende links schneidende Extraktionsschraube eingedreht werden.

Die Extraktionsschraube muss sich linksdrehend eingraben. Unser Trick ist, dass gleichzeitig oder in knapp aufeinander folgenden Intervallen, d. h. nach wenigen Graden Drehung, auf den Griff des Gewindebolzens leichte Hammerschläge ausgeübt werden. Egal wie, man muss mit jedem Drehen das Gefühl haben, dass sich die linksdrehende Extraktionsschraube weiter vorarbeitet und eingräbt. Die Extraktionsschraube muss in Durchmesser und Länge so dimensioniert sein, dass der Konus nicht auf den „Boden“ des Inbus aufläuft (Abb.5). Der Extraktionsbolzen kann dann nicht weiter vorwärts laufen.

Es empfiehlt sich, die Extraktionsschraube an einer bereits ausgedrehten Schraube abzumessen. Es sollte vermieden werden, dass ein „zu kleiner“ Konus verwendet wird. Der gerade nicht zu große Bolzen ist der richtige.

Beachte, dass das „Screw Removal Set“ immer gut mit verschiedenen Extraktionsbolzen bestückt ist.

- Es ist nicht einfach, die Ausdrehkraft so zu dosieren, dass der Extraktionsbolzen nicht abbricht. Manchmal lässt 


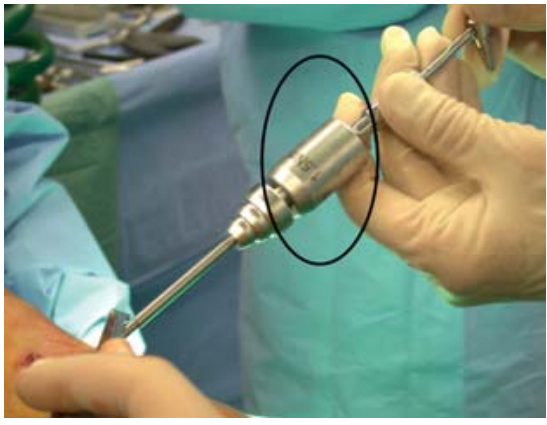

Abb. 1 Kopfverriegelte winkelstabile Schrauben mit Gewinde sollten mit dem passenden Drehmoment angezogen werden, unabhängig davon, ob mit Hand oder mit Maschine.

sich dies nicht vermeiden (s.u.). Eine gewisse Drehmomentbegrenzung wird durch den T-Griff mit Schnellkupplung erreicht. $\mathrm{Ab}$ einer gewissen Kraft beginnt sich der Schaft dieses TGriffes zu verwinden. Im Grunde genommen ist dies ein Zeichen dafür, dass mehr Kraftanwendung eine Gefahr für den Extraktionsbolzen darstellt.

- Mutige Chirurgen können dann statt des T-Griffes mit Schnellkupplung die Extraktionsschraube mit dem Universalbohrfutter greifen. Dann muss aber mit größter Vorsicht links gedreht werden.

Diesen Trick bzw. Schritt vorwärts sollte auch nur jener durchführen, dem dann das Aufbohrset mit Carbide-Bohrern und „Drill Suction Device“ zur Verfügung steht (s.u.).

Nur mit diesen Instrumenten im Hintergrund sollte man das Wagnis des Instrumentenbruchs im Inbus eingehen.

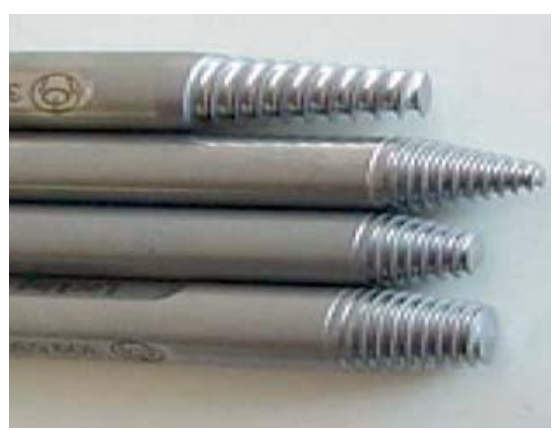

Abb. 4 Linksschneidende Extraktionsschrauben.

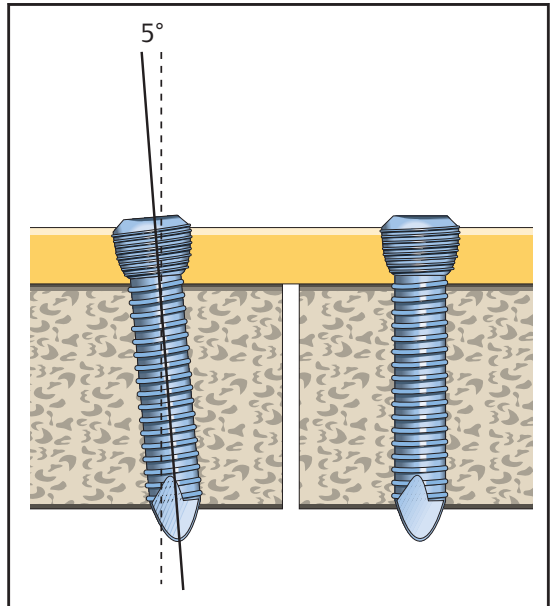

Abb. 2 Monoaxiale Schrauben (z.B. beim LCP-System, LISS-System u.ä.) sollten genau orthograd im rechten Winkel gebohrt und eingebracht werden. Wenn das Gewinde des Kopfes nicht genau in das Gewinde des Plattenlochs hineinläuft, werden Blockierungen provoziert.

- Wenn eine Schraube so blockiert oder zerstört ist, dass die Entfernung mit der Extraktionsschraube nicht möglich ist, muss der Kopf so aufgebohrt werden, dass er sich vom Schaft trennt (Abb.6).

Gleiches gilt für den Fall, dass ein Instrument, ob Schraubendreher oder Extraktionsbolzen, im Inbus abbricht (Abb. 7).

Instrumentenstahl lässt sich natürlich schwerer aufbohren als das Titan oder Stahl der Schrauben.

- Das Aufbohren ohne adäquate Bohrer und Hilfsmittel ist ein großes Problem.

- Zum Aufbohren stehen jetzt neue Instrumente zur Verfügung.

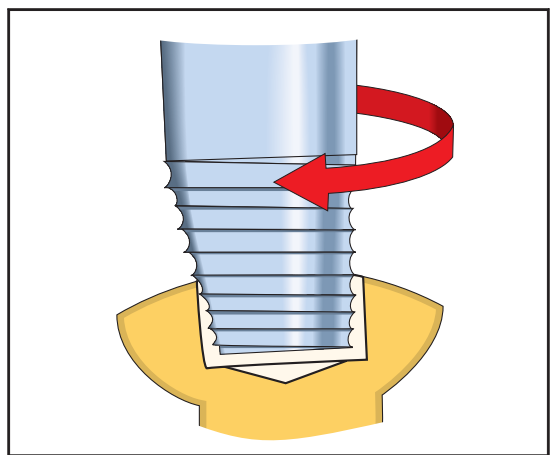

Abb. 5 Die linksdrehende Extraktionsschraube muss genau den richtigen Durchmesser und Länge haben. Das linksdrehende Gewinde muss sich in den Innendurchmesser des Inbus oder des ausgeleierten Inbus eindrehen. Auf der anderen Seite muss er kurz genug sein, damit die Spitze nicht auf den „Boden“ aufläuft.

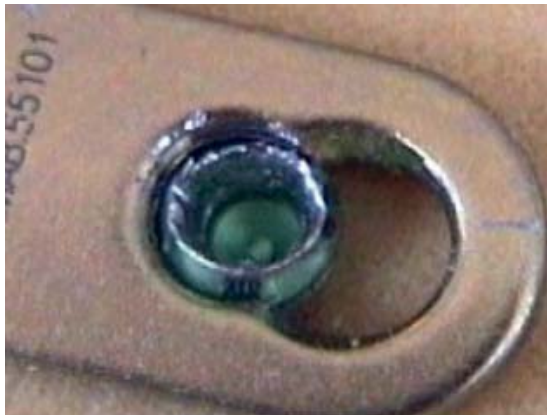

Abb. 3 Ausgeleierter Inbus.

Zum Aufbohren sollten Carbide-Bohrer verwendet werden (Abb.8). In der Industrie haben sich diese Bohrer und Fräswerkzeuge aus der Carbidelegierung schon lange bewährt. Uns stehen jetzt 4,0- und 6,0-Carbide-Bohrer zur Verfügung. Diese sind aus fertigungstechnischen Gründen kurz. Bei Bedarf können sie mit einer „Verlängerung für Schraubendrehereinsatz" verlängert werden. Damit hat man dann einen ausreichend langen Bohrer.

- Daneben können besonders gehärtete HSS-Bohrer verwendet werden. Bei aller Diskussion um den besten Bohrer im Einzelfall hat sich beim Autor und den Mitglieder der MIO-Gruppe der Carbide-Bohrer als bester und neuer „Allroundbohrer“ bestens bewährt. Diese Carbide-Bohrer sind für den Einmalgebrauch gedacht. Bei der Sterilisation können sie Flugrost verursachen. Auf der anderen Seite ist es so, dass nach der schweren Arbeit des Aufbohrens Carbide-Bohrer und HSSBohrer immer etwas abgenutzt sind.

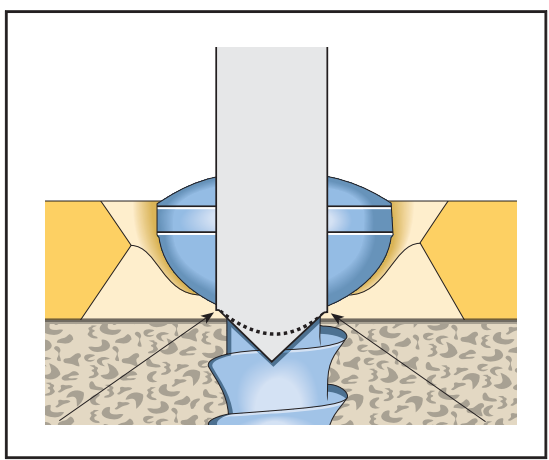

Abb. 6 Der Kopf muss mit einem Bohrer so aufgebohrt werden, dass der Außendurchmesser dieses Bohrers etwas größer ist als der Kerndurchmesser des Schaftes. Dann schneidet der Bohrer den Kopf zirkulär vom Schaft ab. 


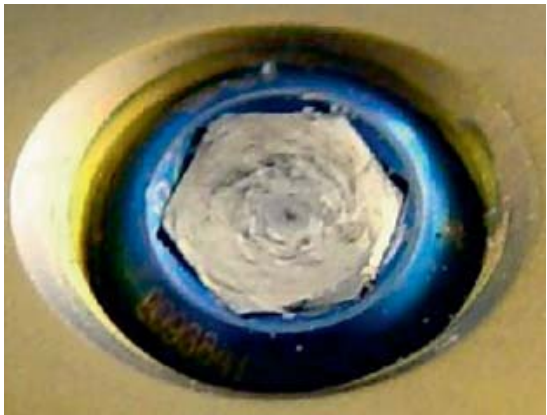

Abb.7 Abgebrochener Schraubenzieher in einem Inbus.

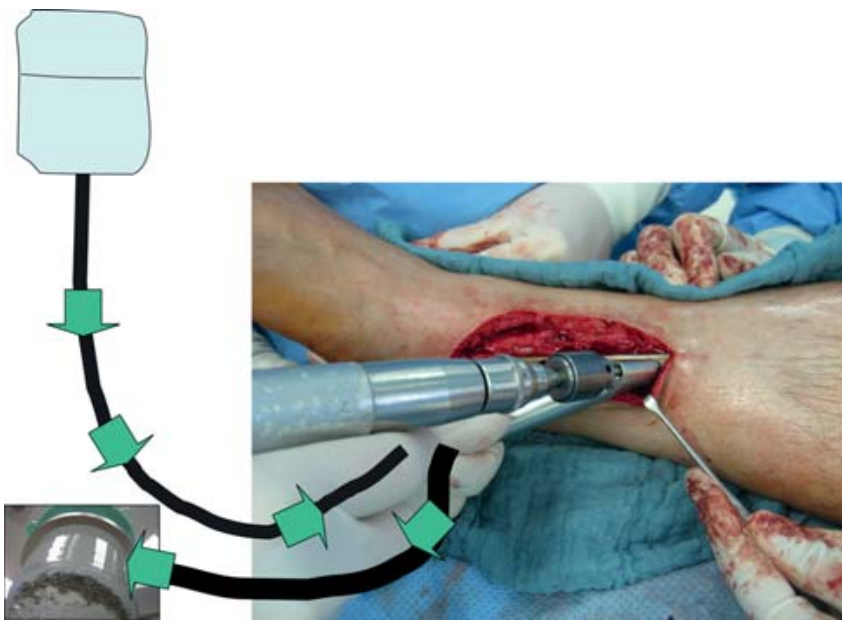

Für die nächste Metallentfernung sollte zur Problembeherrschung wieder ein frischer Bohrer zur Verfügung stehen. Allein die Zeitersparnis rechtfertigt die Kosten.

Regeln zur problematischen Metallentfernung von Schrauben:

- Linksgewindigen Extraktionsbolzen verwenden.

- Darf nicht auf dem Boden aufstoßen.

- Muss gut fassen.

- Wenn das nicht hilft:

Aufbohren mit neuen Carbide-Bohrern.

Das Spül-Saug-Instrumentarium ist für das Aufbohren sehr zu empfehlen.

Seine Funktion ist (Abb. 9):

- Bohren über eine Bohrbüchse, die einen Spülzulauf hat.

- Die Bohrung wird gekühlt und geschmiert.

- Alle Metallspäne werden rückstandslos abgesaugt.

- Die Bohrung wird durch keine Späne behindert.

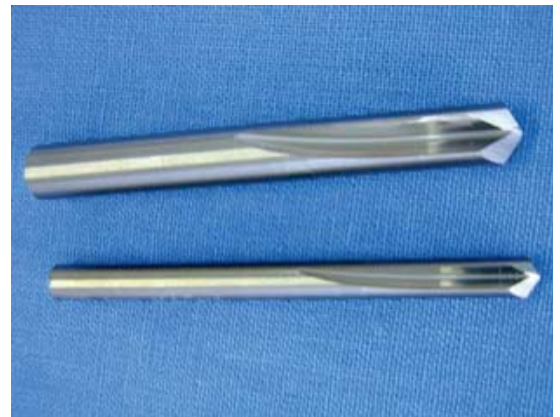

Abb. 8 Carbide-Bohrer in 2 Größen. In der Industrie haben sich die Carbide-Bohrer schon lange bewährt. Es gibt sie dort in einer großen Vielfalt. Für uns stehen mehrere passende Größen zur Verfügung.

Abb. 9 Das SpülSaug-System im Einsatz beim Aufbohren eines Schraubenkopfs. Der Bohrer wird durch eine Führungshülse geführt. Diese wird auf der einen Seite gespült und die Bohrung damit gekühlt. Gleichzeitig werden alle Späne aus der Bohrung befreit. Die Späne stören den Bohrvorgang nicht. Gleichzeitig werden alle Metallspäne sicher und ohne, dass solche Metallspäne ins und ans Gewebe dringen, abgesaugt.

- Ins Gewebe werden keine Späne verschleppt!

- Das Spülsauginstrument ist beim Aufbohren sehr hilfreich.

- Die Bohrung geht besser.

- Wenn alle Schrauben herausgedreht und/oder die Schraubenköpfe abgebohrt sind, können die Platten entfernt werden.

- „Spezialinstrumente“ (konventionell [Abb. 10] oder unkonventionell [Abb. 11] können hilfreich sein.

- Schraubenschäfte können auch belassen werden. Wenn sie entfernt werden sollen:

- Wenn sich die Schraubenschäfte nicht fassen lassen, müssen sie mit einem Hohlfräser überbohrt werden. Der Innendurchmesser soll dem Außendurchmesser der Schraube entsprechen. Dieser Hohlfräser ist linksdrehend $\mathrm{zu}$ verwenden. Manchmal kommt der Schraubenschaft dann mit diesem Hohlfräser heraus. Im Regelfall muss mit einem linksdrehenden Extraktionsbolzen (links schneidendes Innengewinde) der Schraubenschaft entfernt werden (Abb. 12).

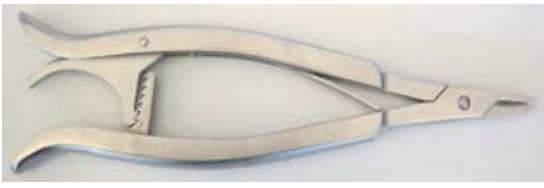

Abb. 10 Kräftige, aber trotzdem schlanke Extraktionszange mit kräftiger Ratsche, damit man beim Ausdrehen nicht selbst halten muss.

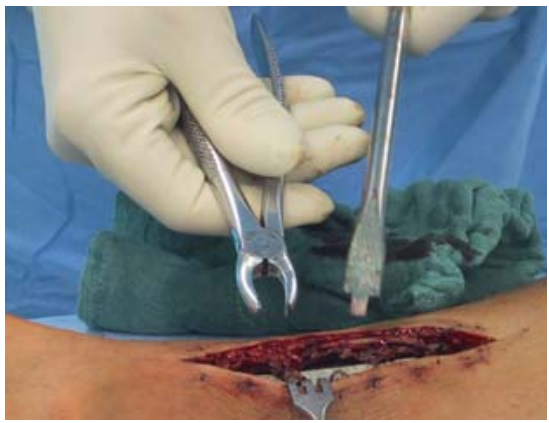

Abb. 11 Spezialinstrumente können helfen, z. B. eine Backenzahnzange der Zahnärzte.

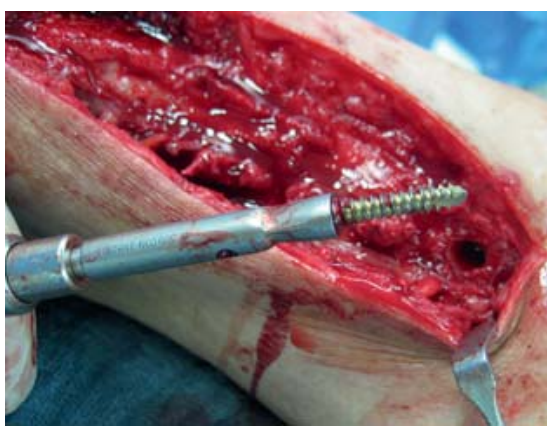

Abb. 12 Linksdrehender Hohlfräser und linksdrehender Extraktionsbolzen helfen beim Entfernen eines Schraubenschafts, dessen Ende „unter der Knochenoberfläche“ liegt.

- Die Prozedur des Überfräsens sollte nur verwendet werden, wenn das große Loch in der zugewandten Kortikalis toleriert werden kann. Oder wenn es notwendig ist, auch den letzten Schraubenrest aus dem Schaft zu entfernen (z.B. bei Infekt oder wenn der Schaft für eine Prothese oder einen Marknagel frei sein muss).

\section{Pathway}

- Ganz wichtig ist eine geordnete Vorwärtsstrategie und den Pathway einzuhalten. Die Instrumente müssen bereitliegen. 


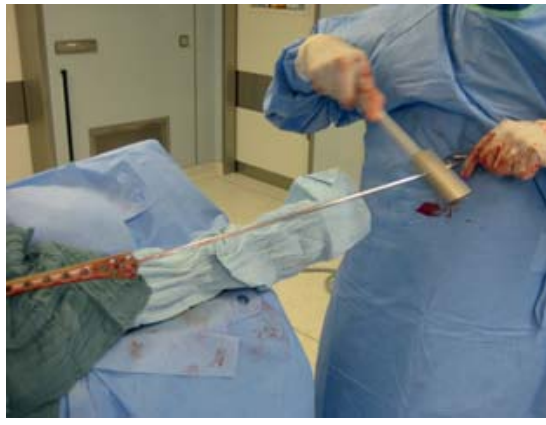

Abb. 13 Minimalinvasiv eingeschobene Platten können minimalinvasiv in Gegenrichtung ausgezogen werden. Statt zu ziehen und zu wackeln, hilft ein „alter“ Küntscher-Haken. Dieser wird in das erste erreichbare Loch eingehängt. Mit einem kräftigen Schlag des Schlitzhammers werden die Knochenbrücken „geknackt“ und die Platte kann gezielt in kleinen Schritten über Schläge herausgezogen werden.

Der Pathway sieht im Grunde genommen so aus:

- Inbus ganz frei machen.

- Besten Schraubendreher schon beim 1. Andrehen verwenden.

- Bei ausgeleiertem Kopf sofort zur passenden links drehenden konischen Extraktionsschraube greifen.

- Wenn das nicht geht oder die Extraktionsschraube oder Schraubenniete abbricht, sofort zum Aufbohren schreiten und zwar mit Carbide-Bohrer und Drill Suction Device.

Wenn diese Schritte eingehalten werden, ist das Entfernen einer blockierten oder ausgeleierten Schraube weder technisch noch zeitlich ein Problem.

\section{Platten}

Wenn alle Schrauben entfernt sind, kann die Platte entfernt werden.

Bei offenen Zugängen macht dies im Regelfall keine Probleme. Kalluswülste oder -stege, welche über die Platte gewachsen sind, müssen manchmal entfernt werden.
Tipps und Tricks für die Entfernung von konventionell oder besonders minimalinvasiv eingeschobenen Platten:

- Befreiung der Platte mit einem Meißel im Insertionsbereich.

- Ein Ausziehversuch, wenn das nicht geht.

- Befreiung der Platte mit einem Meißel, welcher genau die gleiche Breite hat wie die Platte auf der Platte hat.

- Verwendung von rechtwinkligen Meißeln, um an der Kante entlangzufahren.

- All diese Meißel sollten nur vorsichtig vorgeschoben oder mit leichten Hammerschlägen vorgetrieben werden.

Beim Schieben kann es passieren, dass bei plötzlichem Überwinden eines Widerstands der Meißel weiter vorrutscht als gewünscht wurde. Durch leichte Hammerschläge ist die Strecke des Vorrutschens kontrolliert limitiert.

Für das Herausziehen der Platten hat sich in unserer Hand ein „alter“ KüntscherAusziehhaken mit langem Schaft und Ausschlag für einen Schlitzhammer bewährt. Dieser Haken wird in das 1. Loch der Platte eingehängt. Ein Gewebeschutz wird als schiefe Gleitebene daruntergelegt. Mit leichten Hammerschlägen kann nun die Platte gelöst und schrittweise herausgezogen werden.

- Sollte einmal ein kräftiger Hammerschlag zum Überwinden eines Widerstands notwendig sein, empfiehlt es sich, den Griff des Ausziehhakens gegen den eigenen Bauch abzustützen, um dann einen kräftigen Schlag auszuüben. Dadurch wird erreicht, dass die Vorwärtstendenz der Platte sofort wieder kontrolliert gestoppt wird.

- Wenn „minimalinvasiv“ herausgezogen wird, muss in jedem Fall kontrolliert werden, ob es aus dem Plattenlager und dem gesamten Kanal des ehemaligen Implantats zu Blutungen kommt.

- Eine weitere Möglichkeit, eine Platte zu lösen, besteht darin, sie um die Längsachse zu kippen. Hierzu wird ein geeignetes Instrument in eines der Löcher eingebracht und dann um 10 bis $30^{\circ}$ in beide Richtungen gekippt.
Wenn dann Weichteilbrücken und Knochenbrücken aufgebrochen sind, lässt sich im Regelfall die Platte mit oder ohne Haken gut herausziehen.

Stichworte zur leichteren Entfernung der Platten:

- Vorsichtige, aber ausreichende Befreiung der Platte.

- Ausziehen/Schlagen mit „alten“ Küntscherhaken.

- Queres Kippen der Platte.

\section{Fazit}

Das Problem bei der Entfernung von Schrauben und Platten sollte konsequent mit geeignetem Werkzeug angegangen werden.

Das beste Instrument ist gerade gut genug.

Alle Instrumente sollten vorhanden sein.

Wenn der „Pathway“ eingehalten wird, ist auch die „problematische“ Metallentfernung problemlos möglich.

\section{Literatur}

${ }^{1}$ Höntzsch D. Implantatentfernung. Unfallchirurg 2012; 115: 290

${ }^{2}$ Höntzsch D, Stuby FM. Von Platten und Schrauben. Tipps und Tricks für die problematische Metallentfernung. Unfallchirurg 2012; 115: 291-298

${ }^{3}$ Schütz M, Müller M, Krettek C et al. Minimally invasive fracture stabilization of distal femoral fractures with the LISS: a prospective multicenter study. Results of a clinical study with special emphasis on difficult cases. Injury 2001; 32 (Suppl. 3): SC48-SC54

4 Rüedi TP, Buckley R, Moran CG. AO Principles of Fracture Management. New York: Thieme; 2007: 947

Prof. Dr. med. Dankward Höntzsch Leitender Arzt

Abteilung für Medizin-Technische Entwicklungen BG-Unfallklinik Tübingen

Schnarrenbergstraße 95

72076 Tübingen

hoentzsch@t-online.de 